\title{
COMMENT
}

\section{Commentary: Bench to bedside-one step closer?}

\author{
Richard P. Wennberg ${ }^{1}$ \\ Pediatric Research (2018) 84:483-484; https://doi.org/10.1038/s41390-018-0057-1
}

\section{COMMENTARY}

Current guidelines for treating jaundiced newborns are based on limited observational evidence most of which was acquired 3-4 decades ago. As such, evidence supporting current guidelines for jaundice management might not reflect populations cared for today, especially for premature infants. Because of conservative guidelines, close monitoring, and the availability of "safe" and effective phototherapy, acute BIND has become a rare event. Thus, the ability to evaluate new technologies aimed at improving risk assessment is limited. In this issue, Ahlfors and colleagues ${ }^{1}$ offer an approach to expand our assessment of risk within the constraints of existing guidelines.

Since the early 1960's several bilirubin-albumin binding tests have been proposed to either estimate the maximum amount of bilirubin that can be tightly bound to albumin ("binding capacity") or measure free bilirubin (Bf). There is a strong scientific rationale for measuring free bilirubin (Bf) based on accepted pharmacokinetic principles:

1. Only free (unbound) bilirubin (Bf) will cross the blood brain barrier.

2. Albumin has at least two binding sites for bilirubin, shared with potentially competing compounds such as fatty acids and several drugs. The relationships of total plasma bilirubin (TB), Bf, and albumin can be described by the law mass action. $^{2}$

3. The second, lower affinity, binding site becomes increasingly important as the molar ratio bilirubin/albumin (TB/A) exceeds about 0.6 , and provides substantial buffering to TB as the TB/A molar ratio approaches and exceeds 1.0

4. Recent clinical studies indicate that most term infants do not develop residual brain damage or hearing loss unless the TB level is $>30 \mathrm{mg} / \mathrm{dL}^{3-5}$ or when the TB/A ratio approaches or exceeds 1.0. In one study, ${ }^{5}$ several newborns with TB/A's as high as 1.2 did not develop BIND, and determining TB/A did not improve the prediction of acute BIND over TB alone. This suggests that either the quality of binding or susceptibility of brain is important in differentiating risk at a high TB or TB/A.

5. "Binding capacity" (usually reported at a $T B / A \leq 1.0$ ) is a commonly misused term that has meaning only to systems with limited sensitivities for measuring Bf (e.g., Sephadex filtration, front face fluorometry) and cannot be translated easily into quantitative pharmacokinetic models describing drug or bilirubin transport and toxicity. At clinically observed concentrations, albumin has no maximum binding capacity for bilirubin. From the perspective of bilirubin transport in blood, the main determinants of bilirubin distribution in the body are the concentration of plasma albumin and the quality of binding.
Ahlfors et al. ${ }^{1}$ provide a new approach to characterize binding (and relative risk for $A B E$ ) without performing an extensive TB/albumin titration. Sufficient bilirubin is added to a patient's plasma sample to exceed the intended intervention TB. By measuring $B f$ in the original and enriched sample and adjusting the mass action formula for two unknowns, they calculate an arbitrary endpoint TBmax (not a binding capacity). An apparent binding constant $\mathrm{K}$ is determined from the quasi-mass action formula using TBmax, TB, and $\mathrm{Bf}$ (from either titration point). The expected Bf at a given intervention threshold TB (e.g., $25 \mathrm{mg} / \mathrm{dL}$ ) is then estimated from the same formula using the threshold $T B, K$, and TBmax. Since $\mathrm{K}$ is a composite of first and second site binding constants over the 2 point TB range, the accuracy of the calculated $\mathrm{Bf}$ at the prescribed threshold will depend on its position within that range.

An alternative approach to predicting $T B / B f$ relationships was proposed nearly 40 years ago, ${ }^{6}$ comparing log Bf and TB in plasma enriched to provide 2 or more TB concentrations. The investigators observed a linear relationship between log Bf and TB (plotted on semi-log paper); the slope of the line reflected the quality of binding. Predictions of $\mathrm{TB} / \mathrm{Bf}$ relationships using semi-log analysis and Scatchard plot analysis were identical. Either approach can be used to estimate Bf at a given TB (e.g., a proposed intervention guideline) or, conversely, estimate an intervention TB at a given threshold Bf.

How should this information to be applied? The authors propose obtaining population data to define the range of $\mathrm{Bf}$ occurring at risk adjusted TB thresholds (e.g., AAP guidelines). The quality of binding of a given patient can then be compared with that of the general population. The actual risk for an adverse outcome is not addressed, but knowing the comparative risk based on quality of binding may provide support for aggressive or observational management decisions. It is possible, maybe likely, that no cases of BIND will occur within the established population range of $\mathrm{Bf}$, but that in itself will provide important information regarding safe $\mathrm{Bf}$ thresholds.

The low incidence of BIND in affluent countries ironically inhibits development of improved, possibly less aggressive approaches to managing neonatal jaundice, but hyperbilirubinemia remains a major cause of deafness, cerebral palsy, and death in many low-middle income countries. ${ }^{4,5}$ While working to correct the structural and behavioral underpinnings of these disasters, the approach recommended by Ahlfors et al. could be applied to define comparative risks in populations which are indeed at risk, providing the wished for evidence-based thresholds without resorting to comparative "risks" devoid of outcome data.

'Department of Pediatrics, University of Washington, Seattle, USA

Correspondence: Richard P. Wennberg (rpwennberg@hotmail.com)

Received: 10 April 2018 Accepted: 1 May 2018

Published online: 12 September 2018 


\section{ADDITIONAL INFORMATION}

Competing interests: The authors declare no competing interests.

Publisher's note: Springer Nature remains neutral with regard to jurisdictional claims in published maps and institutional affiliations.

\section{REFERENCES}

1. Ahlfors, C. E., Bhutani, V. K., Wong, R. J., Stevenson, D. K. Bilirubin binding in jaundiced newborns: from bench to bedside? Pediatr Res (2018) https://doi.org/ 10.1038/s41390-018-0010-3 (Epub ahead of print).

2. Jacobsen, J. Binding of bilirubin to human serum albumin-determination of the dissociation constants. FEBS Lett. 5, 112-114 (1959).
3. Kuzniewicz, M. W. et al. Incidence, etiology, and outcomes of hazardous hyperbilirubinemia in newborns. Pediatrics 134, 504-509 (2014).

4. Gamaleldin, R. et al. Risk factors for neurotoxicity in newborns with severe neonatal hyperbilirubinemia. Pediatrics 28, e925-e931 (2011).

5. Iskander, I. et al. Serum bilirubin and bilirubin/albumin ratio as predictors of bilirubin encephalopathy. Pediatrics 134, e1330-e1339 (2014).

6. Wennberg, R. P., Rasmussen, L. F., Ahlfors, C. E. \& Valaes, T. Mechanized determination of the apparent unbound unconjugated bilirubin concentration in serum. Clin. Chem. 25, 1444-1447 (1979).

7. Diala, U. M., et al. Patterns of acute bilirubin encephalopathy in Nigeria: a multicenter pre-intervention study. J Perinatol (2018) (Epub ahead of print). 